\title{
Ligand-Induced Shape Transformation of PbSe Nanocrystals
}

\author{
Joep L. Peters, ${ }^{\dagger}$ Karel H. W. van den Bos, ${ }^{\ddagger}$ Sandra Van Aert, ${ }^{\ddagger}$ Bart Goris, ${ }^{\ddagger}$ Sara Bals, ${ }^{\ddagger}$ \\ and Daniël Vanmaekelbergh* $*+$ (i)
}

${ }^{\dagger}$ Debye Institute for Nanomaterials Science, University of Utrecht, Utrecht, The Netherlands

${ }^{*}$ EMAT, University of Antwerp, Antwerpen, Belgium

\section{Supporting Information}

\begin{abstract}
We present a study of the relation between the surface chemistry and nanocrystal shape of $\mathrm{PbSe}$ nanocrystals with a variable $\mathrm{Pb}$ to-Se stoichiometry and density of oleate ligands. The oleate ligand density and binding configuration are monitored by nuclear magnetic resonance and Fourier transform infrared absorbance spectroscopy, allowing us to quantify the number of surface-attached ligands per NC and the nature of the surface- $\mathrm{Pb}$-oleate configuration. The threedimensional shape of the PbSe nanocrystals is obtained from high-angle annular dark field scanning transmission electron microscopy combined with an atom counting method. We show that the enhanced oleate capping results in a stabilization and extension of the $\{111\}$ facets, and a crystal shape transformation from a truncated nanocube to a truncated octahedron.
\end{abstract}

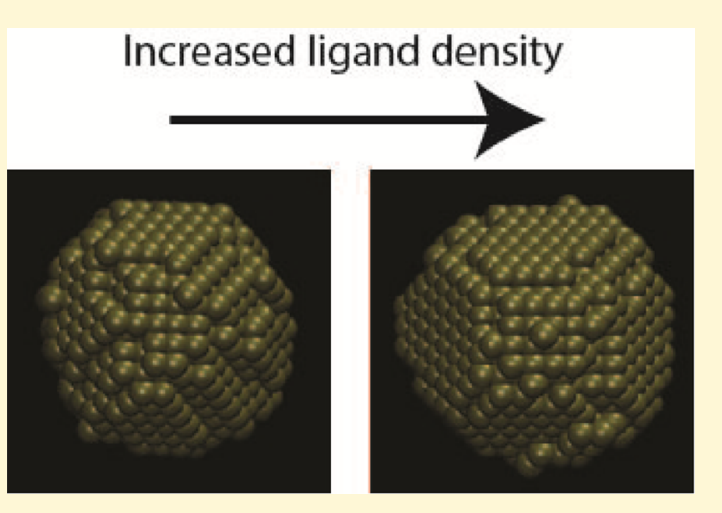

\section{INTRODUCTION}

Nanometer-sized crystals (NCs) of the lead chalcogenide family continue to intrigue researchers for several reasons. First, they show bright exciton luminescence in the near infrared (IR) that can be tuned over a broad energy range by the crystal dimensions. ${ }^{1}$ Second, lead chalcogenide nanocrystals (NCs) can be self-assembled into thin-film quantum dot solids, in which the quantum coupling between the NCs can be tuned. Thin-film systems based on NCs separated by short ligand molecules are of interest for IR photodetectors ${ }^{2}$ and solar cells. ${ }^{3-5}$ Recently, it has been reported that interfacial ordering and epitaxial attachment resulted in NC monolayers with a square or honeycomb nanogeometry. ${ }^{6,7}$

The energy level structure, photoluminescence, and propensity to form atomically coherent two-dimensional (2D) superstructures depend critically on the size and shape of the lead chalcogenide NCs and on their surface chemistry, as well. $^{8-12}$ It has been established that PbSe NCs that are wellcapped with oleate have an excess of $\mathrm{Pb}$ atoms. ${ }^{13-15}$ In addition, it is theoretically shown that the binding strength and configuration of oleate ligands depend on the type of facet. ${ }^{16,17}$

Lead chalcogenide NCs, with PbSe being of interest here, have a rock salt crystal structure. When we consider the uncoordinated crystal, the $\{100\}$ facets, containing square ordered $\mathrm{Pb}$ and $\mathrm{Se}$, have the lowest surface energy. ${ }^{17-19}$ It is thus expected that $\mathrm{PbSe}$ NCs have a cubic shape, terminated by six $\{100\}$ facets. The Wulff reconstruction is presented in section S1 of the Supporting Information. However, in the case of PbSe capped with oleate ligands, different nanocrystal shapes have been reported ranging from a truncated nanocube (or rhombal cuboctahedron) to a truncated octahedron. ${ }^{6,8,20}$
Hence, besides the $\{100\}$ facets, $\{111\}$ and $\{110\}$ facets are also observed. Moreover, it is not yet clear why different $\mathrm{PbSe}$ NC shapes are observed,and how this is related to the surface chemistry.

Here, we present a study of the relation between the surface chemistry and nanocrystal shape of PbSe NCs $(5.6 \mathrm{~nm})$ capped with oleate ligands. $\mathrm{Pb}$-oleate binding is monitored by Fourier transform infrared (FTIR) absorbance and nuclear magnetic resonance (NMR) spectroscopy, allowing us to quantify the nature of the surface $-\mathrm{Pb}$-oleate configuration and the number of surface-attached ligands per NC. The shape of the NCs in three dimensions is obtained via high-angle annular dark field scanning transmission electron microscopy (HAADF-STEM) combined with an atom counting method that has been established previously. ${ }^{21,22}$ We have studied the relation between the shape and surface chemistry under well-defined chemical conditions. After conventional PbSe synthesis, we have washed the NCs eight times to remove all remaining precursor moieties and reduce the oleate capping to a minimum. Then we gradually added $\mathrm{Pb}$ (oleate) $)_{2}$ to the suspension and monitored the attached oleate capping (by NMR) and the NC shape, as well. Our results show that enhanced oleate capping results in a stabilization and extension of the $\{111\}$ facets and a remarkable transformation of the nanocrystal shape.

\footnotetext{
Received: March 21, 2017

Revised: April 6, 2017

Published: April 6, 2017
} 


\section{RESULTS}

Figure 1A shows an overview of the infrared spectrum of a PbSe nanocrystal suspension in tetrachloroethylene (TCE) that
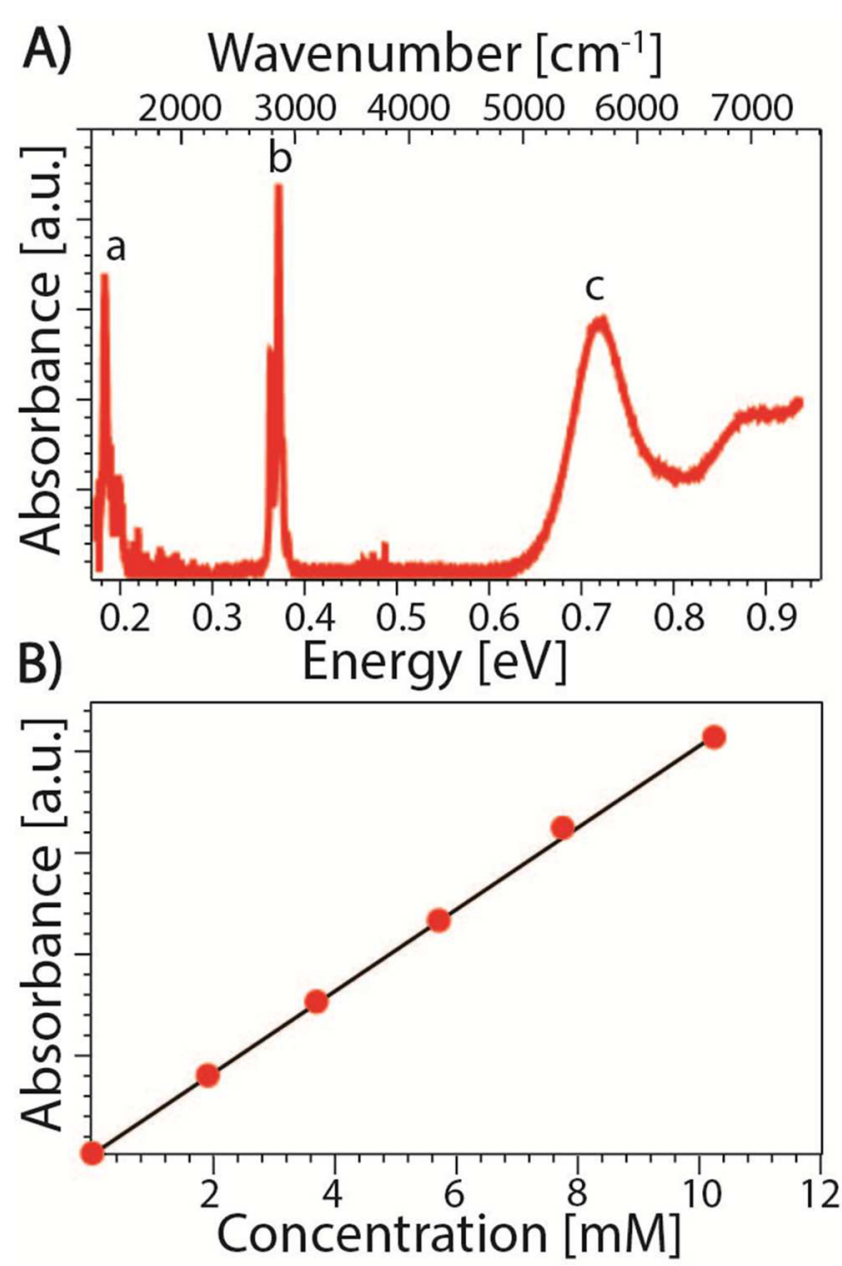

Figure 1. Infrared spectrum of well-washed oleate-capped $\mathrm{PbSe} \mathrm{NCs}$ in tetrachloroethylene. (A) FTIR spectrum of well-washed PbSe NCs $(5.6 \mathrm{~nm})$ dissolved in tetrachloroethylene showing three regions of interest: (a) region of $\mathrm{C}-\mathrm{O}$ vibrations, detailed in Figure 2, (b) a peak due to the $\mathrm{CH}_{2}$ asymmetric stretching vibrations of oleate molecules (attached to the NCs or dissolved), and (c) the IR absorption peak due to the band-edge exciton in the PbSe NCs. From the integration of region $\mathrm{c}$, the concentration of $\mathrm{NCs}$ in the solution can be determined. From the integration of peak $b$, the total concentration of oleate in the suspension (the sum of oleate attached to the NCs and dissolved oleate) can be determined. This is performed by using a calibration curve (panel B), i.e., the integrated absorbance under peak $\mathrm{b}$ vs the concentration of $\mathrm{Pb}$ (oleate $)_{2}$ in a solution containing only $\mathrm{Pb}(\text { oleate })_{2}\left(R^{2}=0.999\right)$.

has been washed eight times with methanol. Washing removes unreacted precursor molecules, excess ligand molecules, and possibly ligand molecules that are only weakly bound to the NCs (see section S2 of the Supporting Information). ${ }^{23,24}$ In Figure $1 \mathrm{~A}$, three regions can be distinguished. The peaks in region a are due to the carboxylic stretching vibrations and will be detailed below. The peaks in region $\mathrm{b}$ are due to the $\mathrm{CH}_{2}$ and $\mathrm{CH}_{3}$ stretching vibrations of the oleate. The IR absorbance in region c, starting with the peak at $0.64 \mathrm{eV}$ and extending to higher energies, reflects the optical transitions in the PbSe NCs. Because of a calibration procedure developed by Moreels et al., ${ }^{13}$ we can use this peak to determine the concentration of $\mathrm{PbSe}$ NCs in the solution.

Using the integrated value under peak $b$, we can determine the total amount of oleate present in the suspension. We therefore calibrated peak b (see Figure 1B). Subsequently, we used the integrated peak value (Figure 1A) to determine the total number of oleate moieties present in the suspension (attached to the NCs and freely dissolved). Combining the integrated values of peaks $b$ and $c$, we can obtain the total number of oleate moieties per PbSe nanocrystal present in the suspension.

We remark here that peaks due to $\mathrm{H}_{2} \mathrm{O}$ and $\mathrm{OH}$ are completely absent in the spectra (see Figure S3), excluding the presence of water, hydroxide, and oleic acid as ligands on our PbSe NCs. A comparison of the FTIR spectra of diphenylphosphine (DPP), trioctylphosphine (TOP), and $\mathrm{Pb}$ (oleate $)_{2}$ with the PbSe NC suspension excludes DPP and indicates that TOP can be present in only small quantities, if it is present at all (see Figure S4). These findings contrast with those reported in the literature, where for $\mathrm{PbS} \mathrm{NCs,} \mathrm{the}$ presence of oleic acid and hydroxide on different facets of the surface of NCs has been reported. ${ }^{17,25}$ We remark here that the hydroxide peak found by Grisorio et al. ${ }^{25}$ cannot be attributed to oxidation of the dots (see Figure S5). As Grisorio et al. claimed, it can be the result from the use of $\mathrm{Pb}$ (oleate) $)_{2}$-hydrate in their advanced synthesis procedure for $\mathrm{PbS}$ nanocrystals. ${ }^{26}$ We also remark that we did not observe hydroxide and oleic acid signals for the NCs synthesized with the method developed by Campos et al. ${ }^{27}$

Now, we focus on the carboxyl stretch vibrations in the energy region between 1350 and $1750 \mathrm{~cm}^{-1}$. A detailed spectrum of the well-washed $\mathrm{PbSe} \mathrm{NCs}$ (Figure 2A-C) shows two broad peaks, reflecting the symmetric $\left(\nu_{1}\right)$ and asymmetric carboxyl stretch $\left(\nu_{2}\right)$ of the oleate moiety (Figure 2B). Figure $2 \mathrm{~A}$ also displays the reference spectra of dissolved $\mathrm{Pb}$ (oleate) $)_{2}$ (green) and oleic acid (blue), indicating that the $\mathrm{Pb}$ (oleate) solution contains oleic acid as an impurity. A spectrum that shows only the features of a pure $\mathrm{Pb}(\text { oleate })_{2}$ solution can be obtained by subtraction (see Figure S6). From comparison of the spectra in Figure 2a, it becomes clear that the spectrum of the well-washed PbSe NCs lacks the peak at $0.212 \mathrm{eV}$, specific for oleic acid, and has a much more pronounced $\nu_{1}$ peak due to the symmetric carboxylic stretch vibration.

Peaks $\nu_{1}$ and $\nu_{2}$ each consist of a doublet (see Figure 2C). For peak $\nu_{1}$, we note the (left) low-energy peak as $\nu_{1, \mathrm{~b}}$ and the one at a higher energy as $\nu_{1, s}$. Notice that for $\nu_{2}$, the lowerenergy peak is denoted as $\nu_{2, s}$ and the high-energy peak as $\nu_{2, \mathrm{~b}}$. The peak splitting suggests that we have two types of $\mathrm{Pb}-$ oleate binding configurations. Cass et al. showed that the inner couple $\left(\nu_{1, \mathrm{~s}}\right.$ and $\left.\nu_{2, \mathrm{~s}}\right)$ can be attributed to an asymmetric chelating bidentate configuration (see Figure $2 \mathrm{E}$ ) if the splitting is between 100 and $125 \mathrm{~cm}^{-1}$. ${ }^{28}$ We find a separation between the inner peaks of $103 \mathrm{~cm}^{-1}$, indicating that the asymmetric $\mathrm{Pb}$-oleate chelating bidentate configuration is one of the dominating configurations. ${ }^{28,29}$ The outer peaks $\left(\nu_{1, \mathrm{~b}}\right.$ and $\left.\nu_{2, \mathrm{~b}}\right)$ point to a bridging configuration of the oleate bound to two neighboring $\mathrm{Pb}$ atoms. The splitting that we observe, $141 \mathrm{~cm}^{-1}$, is in line with such a bridging configuration. ${ }^{30,31}$ The intensity of the symmetric vibration is much larger for NCs than for dissolved $\mathrm{Pb}(\text { oleate })_{2}$. The relative intensity of a vibrational peak increases with the increasing dipole moment of the vibrating moiety. This would mean that the $\mathrm{Pb}$-oleate bidentate complex attached to a facet has a dipole moment 

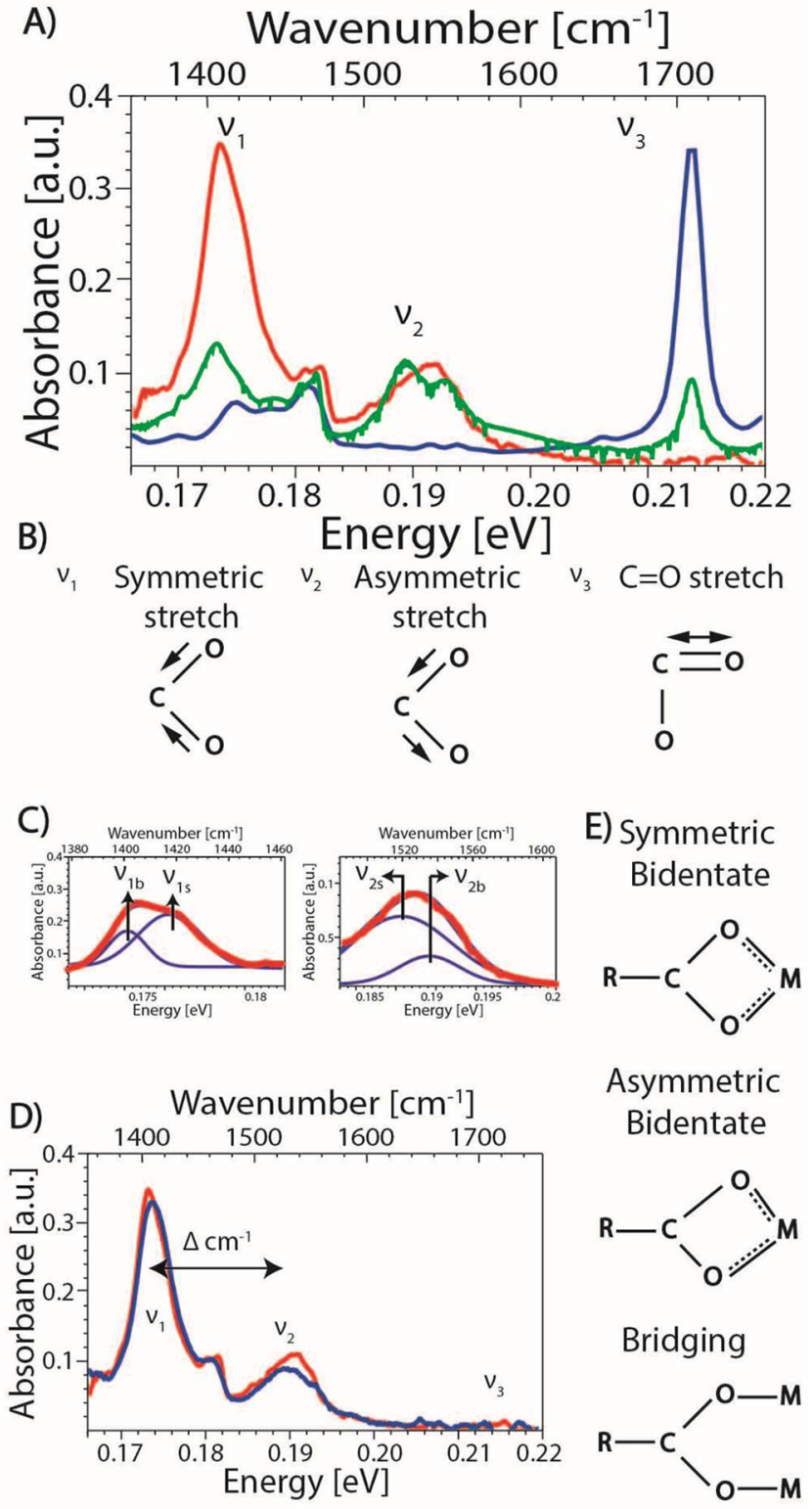

Asymmetric Bidentate

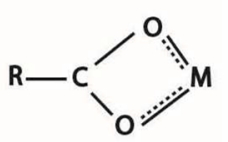

Bridging

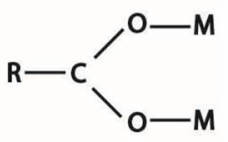

Figure 2. Detailed spectra of the carboxylic region. In panel $A$, the carboxylic regions of washed $\mathrm{NCs}$ (red), $\mathrm{Pb}$ (oleate) ${ }_{2}$ (green), and oleic acid (blue) are shown. The carboxylic vibrations are sketched in panel B. Symmetric and asymmetric carboxylic vibrations $\nu_{1}$ and $\nu_{2}$ in $\mathrm{PbSe} \mathrm{NCs}$ and $\mathrm{Pb}$ (oleate $)_{2}$ are each composed of two peaks. The decomposition and notation are presented in panel $\mathrm{C}$; the meaning is discussed in the text. Panel D shows the carboxylic region for wellwashed NCs (red) and titrated NCs (blue). Panel E presents sketches of the bridging configuration and the (a)symmetric chelating bidentate configurations.

that is stronger than that of the $\mathrm{Pb}$-oleate complexes that form in solution.

Via comparison of the full width at half-maximum (fwhm) of each of the composed peaks, $\nu_{1}$ and $\nu_{2}$, to the corresponding peaks of free $\mathrm{Pb}$ (oleate) $)_{2}$, it is clear that there is a strong broadening of the surface-attached $\mathrm{Pb}$-oleate complex (Table 1). The fwhm of the composed symmetric stretch $\nu_{1}$ increases from 30 to $36 \mathrm{~cm}^{-1}$, while the asymmetric stretch $\nu_{2}$ increases from 56 to $68 \mathrm{~cm}^{-1}$. It is tempting to relate this to the roughness of the facets for which we present evidence below.
Table 1. Distances and Full Widths at Half-Maximum of the Carboxylic Peaks

\begin{tabular}{lcccc}
$\Delta\left(\nu_{1 \mathrm{~b}}-\nu_{2 \mathrm{~b}}\right)$ & $\begin{array}{c}\Delta\left(\nu_{1 \mathrm{~s}}-\nu_{2 \mathrm{~s}}\right) \\
\left(\mathrm{cm}^{-1}\right)\end{array}$ & $\begin{array}{c}\text { fwhm } \nu_{1} \\
\left(\mathrm{~cm}^{-1}\right)\end{array}$ & $\begin{array}{c}\text { fwhm } \nu_{2} \\
\left(\mathrm{~cm}^{-1}\right)\end{array}$ \\
$\mathrm{Pb}(\text { oleate })_{2}$ & 150 & 112 & 30 & 56 \\
well-washed & 141 & 103 & 36 & 68 \\
titrated NCs & 120 & 108 & 35 & 62 \\
\hline
\end{tabular}

Now, we discuss the equilibrium between freely dissolved $\mathrm{Pb}$ (oleate $)_{2}$ and $\mathrm{Pb}$-oleate units at the surface of the $\mathrm{PbSe}$ NCs. With the analysis described above (peak b in Figure 1 referred to the $\mathrm{NC}$ concentration obtained from peak $\mathrm{c}$ in Figure 1) and from NMR results detailed below, we found for the well-washed NC suspension (red curve) an average of 258276 oleates/NC, corresponding to a ligand density of $2.2-2.3$ oleates $/ \mathrm{nm}^{2}$ (assuming a spherical shape). To test if this is really the minimum, a small amount of halide salt dissolved in methanol is added to the suspension. The ligand density can in this way be reduced to 2.0 oleates $/ \mathrm{nm}^{2}$, because of ligand exchange. We did not observe lower densities for colloidally stable PbSe NCs. Next, we performed a titration experiment starting from a suspension of $\mathrm{PbSe} \mathrm{NCs}$, washed eight times, to which we gradually added $\mathrm{Pb}$ (oleate) $)_{2}$. In this titration experiment, we added $\mathrm{Pb}$ (oleate $)_{2}$ to the well-washed suspension and followed the evolution of the ${ }^{1} \mathrm{H}$ NMR signal of the two hydrogen atoms bound to the $\mathrm{C}=\mathrm{C}$ atoms of oleic acid (Figure 3A). It can be seen that because of the large difference in diffusion coefficients, freely dissolved $\mathrm{Pb}$ (oleate) $)_{2}$ (black curve) can be distinguished from the oleate attached to the $\mathrm{NCs}^{24,32}$ (red curve). Via addition of $\mathrm{Pb}$ (oleate) ${ }_{2}$ (colored curves), the spectra combine the contributions of attached and free oleate. They can be quantified by fitting the curves on the basis of the red (only attached oleate) and black (only free oleate) spectra (see Figure S7). Via a reference molecule in a known amount (an internal standard) and the known concentration of NCs, it is also possible to calculate the number of ligands per NC and the ligand density (Figure S8), as demonstrated previously. ${ }^{24,32}$ The results of the titration experiment are presented in Figure 3B, showing that the number of attached oleate moieties first increases to saturate at a maximum of approximately 368 oleates/NC. This must be the maximal oleate ligand coverage possible per nanocrystal, which corresponds to a ligand density of 3.2 oleates $/ \mathrm{nm}^{2}$, in agreement with previous results for as-synthesized NCs. ${ }^{10,32}$

We emphasize that our quantification of the chemisorption process in $\mathrm{Pb}$ (oleate) $)_{2}$ units does not mean that the chemisorbed entities remain preserved as $\mathrm{Pb}$ (oleate) $)_{2}$. In Figure 2 and Table 2, it can be seen that for the titrated NCs the intensity of the outer peaks decreases, indicating relatively less oleate bound in bridging configurations. Moreover, the peak widths are slightly reduced after the titration, indicating that the titration results in facets with less atomic disorder. It will be shown below that $\mathrm{Pb}$ (oleate) $)_{2}$ chemisorption is followed by reconfiguration of the $\mathrm{Pb}$-ligand binding and a transformation of the nanocrystal shape.

We have investigated the three-dimensional (3D) atomic structure of $\mathrm{PbSe} \mathrm{NCs}$ with the minimal and maximal values of oleate capping. Because the NCs in this study are sensitive to the electron beam, high-resolution electron tomography could not be applied to determine their three-dimensional (3D) shape. ${ }^{33-35}$ Therefore, the $3 \mathrm{D}$ atomic structure is extracted from 2D HAADF-STEM projection images, a statistical atom 

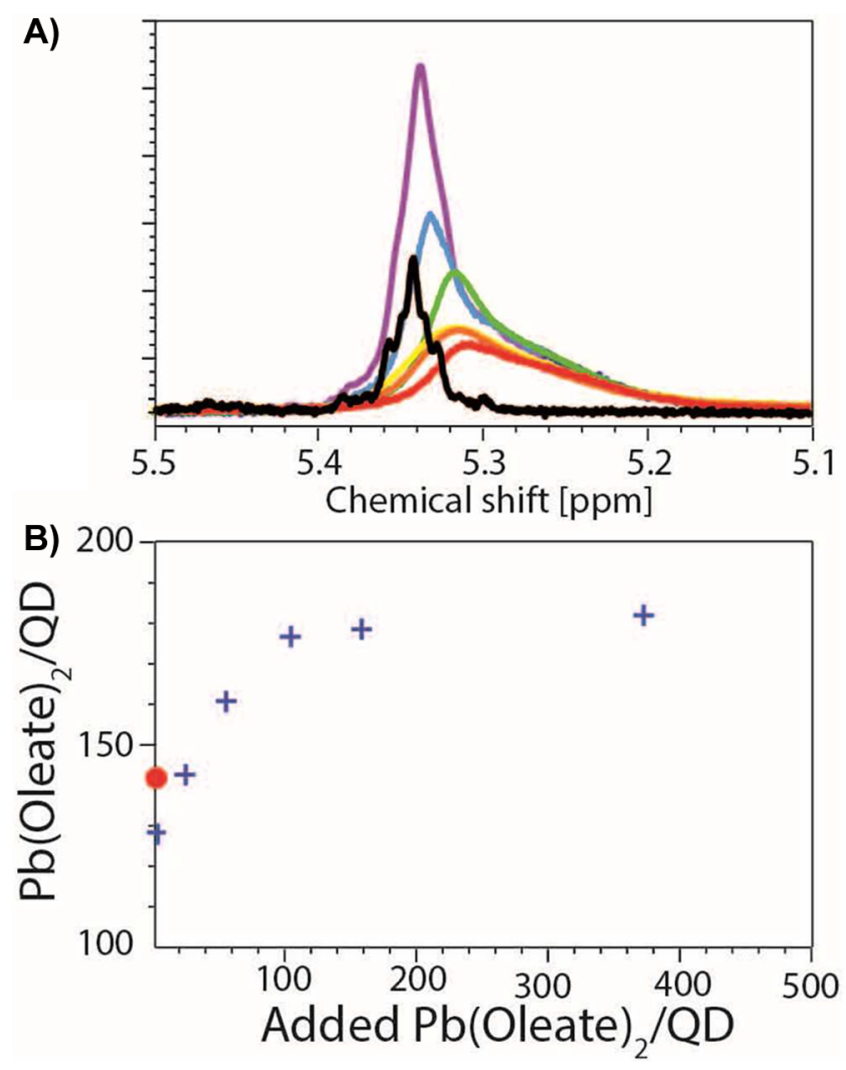

Figure 3. Titration of a well-washed $\mathrm{PbSe} \mathrm{NC}$ suspension with $\mathrm{Pb}$ (oleate) $)_{2}$. A) Close-up of the ${ }^{1} \mathrm{H}$ NMR spectra for the two hydrogen atoms bound to the $\mathrm{C}=\mathrm{C}$ atoms in the oleate. The black spectrum of freely dissolved $\mathrm{Pb}$ (oleate) ${ }_{2}$ and the red spectrum of well-washed $\mathrm{PbSe}$ NCs are normalized on their methyl integral, so spectra at similar oleate concentrations can be compared. The spectrum of well-washed NCs (red) shows a broad feature, indicating all oleate is bound to the surface. The orange, yellow, etc., spectra were obtained during gradual addition of $\mathrm{Pb}$ (oleate) $)_{2}$ to the well-washed $\mathrm{NC}$ suspension. It can be seen that first the magnitude of the broad peak increases (maximum at $5.3 \mathrm{ppm}$ ) while with an increase in the amount of $\mathrm{Pb}$ (oleate) ${ }_{2}$ added the sharp peak at $5.35 \mathrm{ppm}$ also arises. Deconvolution of both peaks (shown in Figure S7) allowed us to quantify the number of attached and freely dissolved $\mathrm{Pb}$ (oleate) $)_{2}$ per $\mathrm{NC}$ during the titration experiment. This is shown in panel B, including the red dot determined via FTIR. The derived ligand densities are colored orange $\left(2.4\right.$ oleates $\left./ \mathrm{nm}^{2}\right)$, yellow $\left(2.5\right.$ oleates $\left./ \mathrm{nm}^{2}\right)$, green $\left(3.2\right.$ oleates $\left./ \mathrm{nm}^{2}\right)$, blue $\left(3.1\right.$ oleates $\left./ \mathrm{nm}^{2}\right)$, and purple $\left(3.2\right.$ oleates $\left./ \mathrm{nm}^{2}\right)$.

Table 2. Percentages of Surface Atoms That Have a Specific Coordination Number That Corresponds to Surface Facets

\begin{tabular}{lcc} 
& low ligand density & high ligand density \\
$\{100\}$ coordination & $12.1 \%(10.7-13.4 \%)$ & $13.6 \%(12.2-15.0 \%)$ \\
$\{110\}$ coordination & $18.3 \%(16.7-20.0 \%)$ & $14.9 \%(13.4-16.3 \%)$ \\
$\{111\}$ coordination & $17.3 \%(15.7-18.9 \%)$ & $21.8 \%(20.1-23.4 \%)$ \\
irregular coordination & $52.3 \%(50.2-54.4 \%)$ & $49.8 \%(47.7-51.8 \%)$ \\
\hline
\end{tabular}

counting procedure and an energy relaxation procedure, developed in previous work (see Figures S9 and S10). ${ }^{21,36,37}$

HAADF-STEM images showing an overview of the particles with minimal and maximal oleate coverage are displayed in panels A and F of Figure 4, respectively. The NCs with a high ligand density assemble into a hexagonal array. HAADF-STEM images acquired using a higher magnification for both samples are presented in panels B and G of Figure 4. Both images show a nanocrystal oriented along a $[110]$ zone axis. These images have been used as input for a statistical atom counting procedure yielding the number of atoms in each projected atomic column (Figure 4C,H). Here, counting results are obtained with $90 \%$ single-atom sensitivity, meaning that for $90 \%$ of all atom columns the number of atoms is correctly assigned, whereas the probability to miscount by \pm 1 atom is $10 \%$.

Starting from these numbers, we can obtain a 3D model for each individual nanocrystal using a Monte Carlo-based energy minimization scheme. ${ }^{37,38}$ We have performed such a procedure for 10 different NCs of both ligand densities, as presented in Figure S9. It can be observed that for the particles with a low ligand density, the crystal facets are rather small and contain several loose atoms. In line with atomic simulations of ligand-free NCs, we attribute this surface roughness to atomic surface reconstructions. ${ }^{18}$ The possibility that the electron beam induces some atom sublimation, however, cannot be excluded, although the exposure time is kept as short as possible. The $3 \mathrm{D}$ shape determination shows that particles with a high ligand density have $\{111\}$ facets that are larger than those of low-ligand density particles. To obtain a prototypical shape of the PbSe NCs, an averaging method was applied that uses the 3D models of the individual NCs as a basis (see Figure S10 for a more detailed explanation of the averaging procedure). The result of this procedure is presented in panels $\mathrm{D}$ and I of Figure 4; it can be observed that the shape of the well-washed PbSe NCs (low level of oleate capping) is a nanocube in which truncation results in the presence of $\{110\}$ and $\{111\}$ facets, besides the $\{100\}$ facets. In addition, there is a considerable degree of atomic disorder present on each of these facets that we attribute to atomic surface reconstructions of the bare crystal facets.

For the NCs with maximal oleate capping, a remarkable transformation of the shape is observed. The $\{111\}$ facets become more dominant at the expense of the $\{110\}$ facets, although the latter do not disappear completely. Thus, the added $\mathrm{Pb}$ (oleate $)_{2}$ ligands stabilize and order the $\{111\}$ facets. The truncated octahedral shape is similar to that found for $\mathrm{PbS}$ crystals $^{17}$ and for as-synthesized PbSe NCs. ${ }^{16,20}$ Idealized models are presented in panels $\mathrm{E}$ and $\mathrm{J}$ of Figure 4 . To quantify the increase in the number of $\{111\}$ facets for the sample with a high ligand density, the coordination number of surface $\mathrm{Pb}$ atoms with other $\mathrm{Pb}$ atoms can be used. This number can be related to the surface types that are present in the final configurations. The results are presented in Table 2 together with the $68 \%$ confidence intervals, indicating that $22 \%$ of the surface atoms belong to a $\{111\}$ facet (coordination number of 9) for the sample with a high ligand density. For the PbSe NCs with a low ligand density, only $17 \%$ of the surface atoms belonged to a $\{111\}$ facet. The number of irregular atoms, not having a $\{100\},\{110\}$, or $\{111\}$ coordination number, decreases from 53 to $49 \%$ with an increase in ligand density. Edge atoms are also included in the irregular category, because these have a lower coordination number. Thus, the real facets are on average two $\mathrm{Pb}$ atoms larger in the two orthogonal directions (see Figure 4) than following from Table 2.

\section{DISCUSSION}

We start with the well-washed PbSe NCs that have a truncated cubic shape with rather rough facets and a minimum of capping ligands. For such a nanocrystal type, the surface energies of the three facets have been calculated by Schapotschnikov et al. ${ }^{18}$ for 

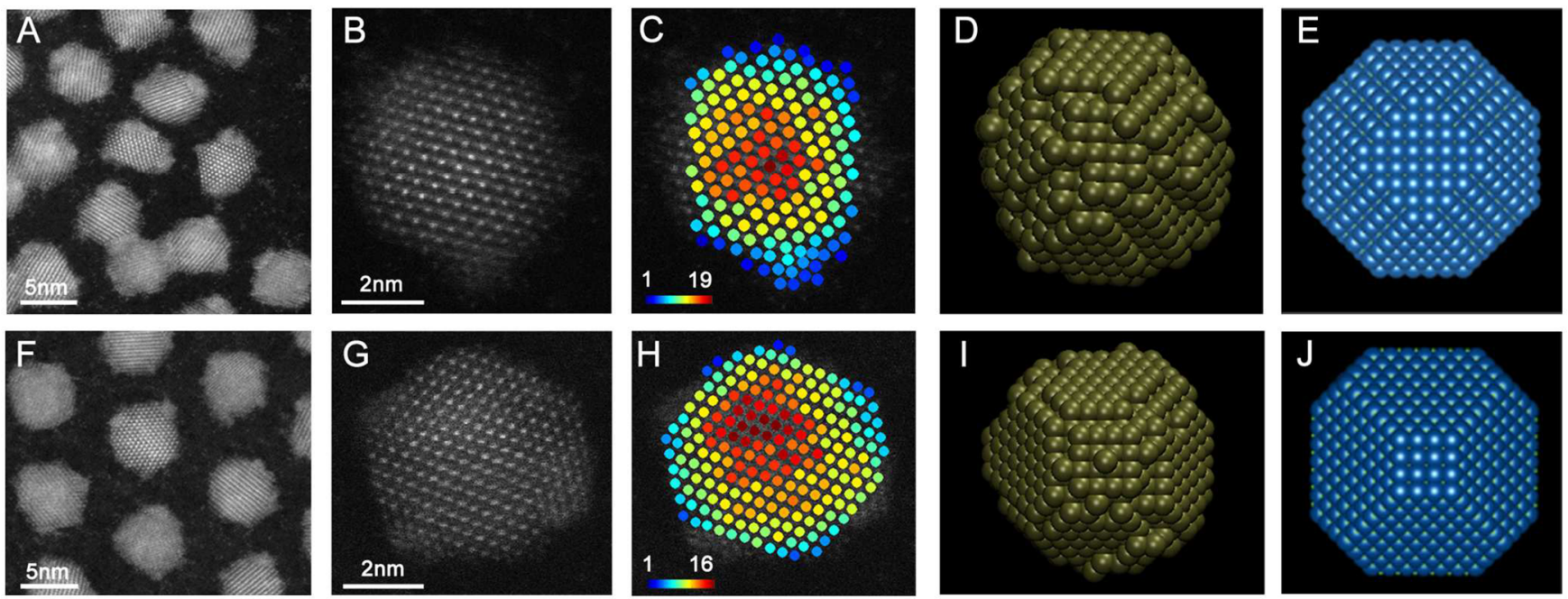

Figure 4. HAADF-STEM study of PbSe NCs with an atom counting technique. The top row (A-E) shows the shape analysis for low-ligand density $\mathrm{NCs}$ and the bottom row (F-J) the maximal ligand densities. Panels A and F are HAADF-STEM images of a group of PbSe NCs, whereas panels B and $\mathrm{G}$ are magnified images for PbSe NCs that are oriented in such a way that the lead atoms are oriented in columns. In panels $\mathrm{C}$ and $\mathrm{H}$, the intensities of the columns are integrated and the numbers of lead atoms in that row are counted and color-coded. Subsequently, 10 NCs of each sample are averaged (see Figure S10 for the procedure) in panels D and I. We propose models E and J for the fitted data shown in panels D and I, respectively. Notice the increased size of the $\{111\}$ facets for the oleate-saturated NCs.

the case in which no capping is present: the $\{100\}$ facets have an energy much lower than that of the $\{110\}$ and $\{111\}$ facets. In fact, naked $\{111\}$ facets have such a high energy that they tend to be reconstructed in simulations: on Pb-terminated $\{111\}$ surfaces, for example, $\mathrm{Pb}$ atoms are partially pulled inward while Se atoms pop up, resulting in a reconstructed rough surface. The well-washed samples have a relatively small number of oleate ligands [258-276 per nanocrystal (see above)]; it is reasonable to assume that they are preferentially bound to $\mathrm{Pb}$ atoms of the $\{111\}$ surfaces to minimize the energy of the entire nanocrystal. The amount of surface lead atoms on the $\{111\}$ crystalline facet can be roughly estimated from the averaged model (Figure $4 \mathrm{E}$ ). This results in $220 \mathrm{~Pb}$ atoms; this number is too small to accommodate all oleate ligands ( 258 or 276 on average), and thus, $\sim 20 \%$ of the oleates is bound the $\{110\}$ or $\{100\}$ surfaces. The ideal position of a $\mathrm{Pb}$-oleate moiety would be in the fcc valley of three Se atoms at the $\{111\}$ facet; the $\mathrm{Pb}$-oleate moieties attached to the $\{110\}$ or $\{100\}$ facets together with surface roughness would explain the broadening of the $\nu_{1}$ and $\nu_{2}$ peaks in the carboxylic region (see Figure 2).

What happens to the well-washed PbSe NCs when $\mathrm{Pb}$ (oleate $)_{2}$ is added to the $\mathrm{PbSe} \mathrm{NC}$ suspension? Initially, the added $\mathrm{Pb}$ (oleate) $)_{2}$ can bind to any Se surface atom, as an $\mathrm{MX}_{2}$ or Z-type ligand. ${ }^{39}$ It has been reported that the (surface) atoms of PbSe nanocrystals have a high mobility, even at room temperature. $^{23,24,40}$ This should allow the chemisorbed Pb(oleate) $)_{2}$ to reconfigure such that the energy of the $\mathrm{PbSe}$ nanocrystals is minimized. We observed that the $\{111\}$ facets become more extended at the expense of the $\{110\}$ facets. This can occur via the following steps. (i) The chemisorbed $\mathrm{Pb}$ (oleate $)_{2}$ is split into a $\mathrm{Pb}$ (oleate) moiety that becomes optimally coordinated in the valley of three Se surface atoms. (ii) The remaining oleate binds to a free $\mathrm{Pb}$ atom at a valley position. This process is presented in a schematic way in Figure 5. The process of $\mathrm{Pb}$ (oleate $)_{2}$ chemisorption, splitting, and stabilization and extension of $\mathrm{Pb}$-oleate-terminated $\{111\}$ surfaces is schematically presented in Scheme 1.

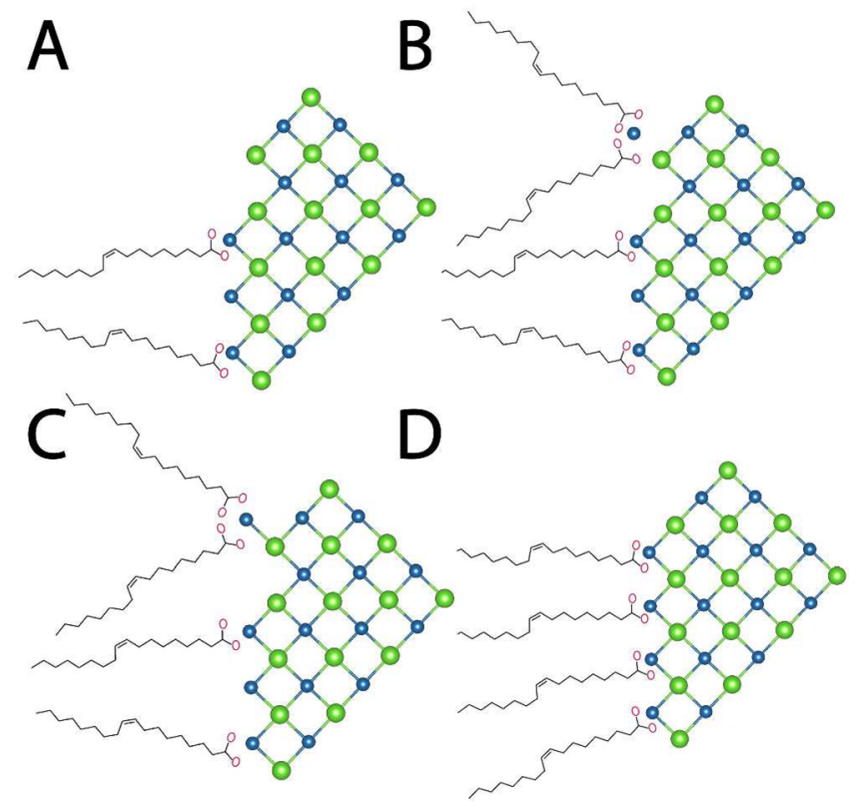

Figure 5. Scheme describing the attachment and integration of a $\mathrm{Pb}$ (oleate) ${ }_{2}$. Attachment of these Z-type ligands on a $\mathrm{PbSe} \mathrm{NC}$ resulting in stabilization of a $\mathrm{Pb}-$ oleate-terminated $\{111\}$ facet. Panel A shows an unsaturated facet, typical for well-washed PbSe NCs. Panels $\mathrm{B}$ and $\mathrm{C}$ show the chemisorbtion of $\mathrm{Pb}(\text { oleate })_{2}$ as a Z-type ligand. Panel $\mathrm{D}$ shows the stabilization of the $\mathrm{Pb}$-oleate-terminated $\{111\}$ facets.

Scheme 1. Chemisorption of $\mathrm{Pb}(\text { oleate })_{2}$ and the Ensuing Reconfiguration

(1) $\left(\mathrm{Pb}_{\mathrm{m}+(\mathrm{y} / 2)} \mathrm{Se}_{\mathrm{m}}\right)(\text { oleate })_{\mathrm{y}}+\mathrm{n} \mathrm{Pb}(\text { oleate })_{2} \rightarrow$ $\left.\left(\mathrm{Pb}_{\mathrm{m}+(\mathrm{y} / 2)} \mathrm{Se}_{\mathrm{m}}\right)(\text { oleate })_{\mathrm{y}}\right)\left(\mathrm{Pb}(\text { oleate })_{2}\right)_{\mathrm{n}}$

(2) $\left.\left(\mathrm{Pb}_{\mathrm{m}+(\mathrm{y} / 2)} \mathrm{Se}_{\mathrm{m}}\right)(\text { oleate })_{\mathrm{y}}\right)\left(\mathrm{Pb}(\text { oleate })_{2}\right)_{\mathrm{n}} \rightarrow$ $\mathrm{Pb}_{\mathrm{m}+(\mathrm{y} / 2)+\mathrm{n}} \mathrm{Se}_{\mathrm{m}}$ (oleate $)_{\mathrm{y}+2 \mathrm{n}}$ 
This formal notation accounts for charge neutrality and mass balance. The reconfiguration results in a stabilization and extension of the $\{111\}$ facets, in agreement with the observation. For the well-washed PbSe samples, $y$ is approximately 184 , including the edges of the $\{111\}$ facets. After chemisorption of the added $\mathrm{Pb}$ (oleate) $)_{2}$ and the reconfiguration process, the maximal excess of $\mathrm{Pb}$ per nanocrystal amounts to $y / 2+n$. The maximal oleate coverage $y+2 n$ is equal to 368 oleates/NC. This can be compared with the number of available $\{111\}$ lead surface positions on the averaged PbSe NCs presented in Figure 4I, being 356. This means that $\mathrm{PbSe}$ nanocrystals with a maximal oleate coverage have almost all oleate ligands attached to the $\{111\}$ facets and its edges.

By the addition of $\mathrm{Pb}$ (oleate) $)_{2}$ to a suspension of wellwashed NCs, the NCs acquire a larger excess of lead atoms, the charge neutrality is preserved, and the ligand stabilization of the $\{111\}$ surfaces is optimized (see Scheme 1 and Figure 5). This is in line with the reduced IR line widths, indicating reduced levels of atomic disorder on the facets and more (a)symmetric carboxylic configurations (see Table 1 and Figure 2D). Moreover, the stabilization and extension of the $\{111\}$ facets result in a shape transformation of the nanocrystal from a truncated cube to a cuboctahedron (truncated octahedron) solely with $\{111\}$ and $\{100\}$ facets. We believe that the final situation that we reach by addition of $\mathrm{Pb}$ (oleate) $)_{2}$ to wellwashed samples is close to that of "as-synthesized" $\mathrm{PbSe}$ nanocrystals.

In summary, we have used IR absorption spectroscopy and $\mathrm{NMR}$ to quantitatively measure the number of $\mathrm{Pb}$ (oleate) ligands per $\mathrm{PbSe}$ nanocrystal. We have shown that $\mathrm{PbSe} \mathrm{NCs}$ can be colloidally stable with a wide range of oleate ligand densities. Our washing and titration cycle suggests that the process of removing and adding oleate ligands is reversible. $\mathrm{Pb}$ (oleate $)_{2}$ chemisorption and surface reconfiguration result in a transformation of the nanocrystal shape from a truncated nanocube with rough surface facets to a truncated octahedron with larger and smooth $\{111\}$ facets.

\section{ASSOCIATED CONTENT}

\section{S Supporting Information}

The Supporting Information is available free of charge on the ACS Publications website at DOI: 10.1021/acs.chemmater.7b01103.

Experimental methods, explanation of the Wulff reconstruction, several supporting FTIR spectra, separation of oleic acid from $\mathrm{Pb}$ (oleate) $)_{2}$, procedure for determining the amount of bound and unbound $\mathrm{Pb}$ (oleate) $)_{2}$ via NMR, and supportive HAADF-STEM data, including a detailed explanation of the averaging procedure (Figure S9) (PDF)

\section{AUTHOR INFORMATION}

\section{Corresponding Author}

*E-mail: d.vanmaekelbergh@uu.nl.

ORCID ${ }^{\circ}$

Joep L. Peters: 0000-0002-3415-648X

Sara Bals: 0000-0002-4249-8017

Daniël Vanmaekelbergh: 0000-0002-3535-8366

Notes

The authors declare no competing financial interest.

\section{ACKNOWLEDGMENTS}

D.V. acknowledges the European Research Council, ERC advanced grant, Project 692691-First Step, for financial support. We also acknowledge the Dutch FOM programme "Designing Dirac carriers in honeycomb semiconductor superlattices" (FOM Program 152) for financial support. The authors gratefully acknowledge funding from the Research Foundation Flanders (G.036915, G.037413, and funding of a Ph.D. research grant to K.H.W.v.d.B. and a postdoctoral grant to B.G.). S.B. acknowledges the European Research Council, ERC Grant 335078-Colouratom.

\section{REFERENCES}

(1) Steckel, J. S.; Coe-Sullivan, S.; Bulović, V.; Bawendi, M. G. $1.3 \mu \mathrm{m}$ to $1.55 \mu \mathrm{m}$ Tunable Electroluminescence from PbSe Quantum Dots Embedded within an Organic Device. Adv. Mater. 2003, 15, 18621866.

(2) McDonald, S. A.; Konstantatos, G.; Zhang, S.; Cyr, P. W.; Klem, E. J. D.; Levina, L.; Sargent, E. H. Solution-Processed PbS Quantum Dot Infrared Photodetectors and Photovoltaics. Nat. Mater. 2005, 4, $138-142$.

(3) Zhang, J.; Gao, J.; Church, C. P.; Miller, E. M.; Luther, J. M.; Klimov, V. I.; Beard, M. C. PbSe Quantum Dot Solar Cells with More than 6\% Efficiency Fabricated in Ambient Atmosphere. Nano Lett. 2014, 14, 6010-6015.

(4) Marshall, A. R.; Young, M. R.; Nozik, A. J.; Beard, M. C.; Luther, J. M. Exploration of Metal Chloride Uptake for Improved Performance Characteristics of PbSe Quantum Dot Solar Cells. J. Phys. Chem. Lett. 2015, 6, 2892-2899.

(5) Lan, X.; Voznyy, O.; Kiani, A.; García de Arquer, F. P.; Abbas, A. S.; Kim, G.; Liu, M.; Yang, Z.; Walters, G.; Xu, J.; Yuan, M.; Ning, Z.; Fan, F.; Kanjanaboos, P.; Kramer, I.; Zhitomirsky, D.; Lee, P.; Perelgut, A.; Hoogland, S.; Sargent, E. H. Passivation Using Molecular Halides Increases Quantum Dot Solar Cell Performance. Adv. Mater. 2016, 28, 299-304.

(6) Evers, W. H.; Goris, B.; Bals, S.; Casavola, M.; De Graaf, J.; van Roij, R.; Dijkstra, M.; Vanmaekelbergh, D. Low-Dimensional Semiconductor Superlattices Formed by Geometric Control over Nanocrystal Attachment. Nano Lett. 2013, 13, 2317-2323.

(7) Boneschanscher, M. P.; Evers, W. H.; Geuchies, J. J.; Altantzis, T.; Goris, B.; Rabouw, F. T.; van Rossum, S. A. P.; van der Zant, H. S. J.; Siebbeles, L. D. A.; Van Tendeloo, G.; Swart, I.; Hilhorst, J.; Petukhov, A. V.; Bals, S.; Vanmaekelbergh, D. Long-Range Orientation and Atomic Attachment of Nanocrystals in 2D Honeycomb Superlattices. Science (Washington, DC, U. S.) 2014, 344, 1377-1380.

(8) Baumgardner, W. J.; Whitham, K.; Hanrath, T. Confined-butConnected Quantum Solids via Controlled Ligand Displacement. Nano Lett. 2013, 13, 3225-3231.

(9) Allan, G. Surface Core-Level Shifts and Relaxation of Group-IV A-Element Chalcogenide Semiconductors. Phys. Rev. B: Condens. Matter Mater. Phys. 1991, 43, 9594-9598.

(10) Choi, J. J.; Bealing, C. R.; Bian, K.; Hughes, K. J.; Zhang, W.; Smilgies, D. M.; Hennig, R. G.; Engstrom, J. R.; Hanrath, T. Controlling Nanocrystal Superlattice Symmetry and Shape-Anisotropic Interactions through Variable Ligand Surface Coverage. J. Am. Chem. Soc. 2011, 133, 3131-3138.

(11) Evers, W. H.; Schins, J. M.; Aerts, M.; Kulkarni, A.; Capiod, P.; Berthe, M.; Grandidier, B.; Delerue, C.; van der Zant, H. S. J.; van Overbeek, C.; Peters, J. L.; Vanmaekelbergh, D.; Siebbeles, L. D. A. High Charge Mobility in Two Dimensional Percolative Networks of PbSe Quantum Dots Connected by Atomic Bonds. Nat. Commun. 2015, 6, 8195.

(12) Kang, I.; Wise, F. W. Electronic Structure and Optical Properties of $\mathrm{PbS}$ and $\mathrm{PbSe}$ Quantum Dots. J. Opt. Soc. Am. B 1997, 14, 16321646.

(13) Moreels, I.; Lambert, K.; De Muynck, D.; Vanhaecke, F.; Poelman, D.; Martins, J. C.; Allan, G.; Hens, Z. Composition and Size- 
Dependent Extinction Coefficient of Colloidal PbSe Quantum Dots. Chem. Mater. 2007, 19, 6101-6106.

(14) Fritzinger, B.; Capek, R. K.; Lambert, K.; Martins, J. C.; Hens, Z. Utilizing Self-Exchange To Address the Binding of Carboxylic Acid Ligands to CdSe Quantum Dots. J. Am. Chem. Soc. 2010, 132, 1019510201.

(15) Owen, J. S.; Park, J.; Trudeau, P.-E. E.; Alivisatos, a. P. Reaction Chemistry and Ligand Exchange at Cadmium-Selenide Nanocrystal Surfaces. J. Am. Chem. Soc. 2008, 130, 12279-12281.

(16) Bealing, C. R.; Baumgardner, W. J.; Choi, J. J.; Hanrath, T.; Hennig, R. G. Predicting Nanocrystal Shape through Consideration of Surface-Ligand Interactions. ACS Nano 2012, 6, 2118-2127.

(17) Zherebetskyy, D.; Scheele, M.; Zhang, Y.; Bronstein, N.; Thompson, C.; Britt, D.; Salmeron, M.; Alivisatos, A. P.; Wang, L.-W. Hydroxylation of the Surface of $\mathrm{PbS}$ Nanocrystals Passivated with Oleic Acid. Science (Washington, DC, U. S.) 2014, 344, 1380-1384.

(18) Schapotschnikow, P.; van Huis, M. A.; Zandbergen, H. W.; Vanmaekelbergh, D.; Vlugt, T. J. H. Morphological Transformations and Fusion of PbSe Nanocrystals Studied Using Atomistic Simulations. Nano Lett. 2010, 10, 3966-3971.

(19) Fang, C.; Van Huis, M. a.; Vanmaekelbergh, D.; Zandbergen, H. W. Energetics of Polar and Nonpolar Facets of PbSe Nanocrystals from Theory and Experiment. ACS Nano 2010, 4, 211-218.

(20) Cho, K. S.; Talapin, D. V.; Gaschler, W.; Murray, C. B. Designing PbSe Nanowires and Nanorings through Oriented Attachment of Nanoparticles. J. Am. Chem. Soc. 2005, 127, 7140-7147.

(21) Van Aert, S.; De Backer, A.; Martinez, G. T.; Goris, B.; Bals, S.; Van Tendeloo, G.; Rosenauer, A. Procedure to Count Atoms with Trustworthy Single-Atom Sensitivity. Phys. Rev. B: Condens. Matter Mater. Phys. 2013, 87, 64107.

(22) Bals, S.; Casavola, M.; van Huis, M. A.; Van Aert, S.; Batenburg, K. J.; Van Tendeloo, G.; Vanmaekelbergh, D. Three-Dimensional Atomic Imaging of Colloidal Core-Shell Nanocrystals. Nano Lett. 2011, 11, 3420-3424.

(23) Hassinen, A.; Moreels, I.; De Nolf, K.; Smet, P. F.; Martins, J. C.; Hens, Z. Short-Chain Alcohols Strip X-Type Ligands and Quench the Luminescence of $\mathrm{PbSe}$ and CdSe Quantum Dots, Acetonitrile Does Not. J. Am. Chem. Soc. 2012, 134, 20705-20712.

(24) Anderson, N. C.; Hendricks, M. P.; Choi, J. J.; Owen, J. S. Ligand Exchange and the Stoichiometry of Metal Chalcogenide Nanocrystals: Spectroscopic Observation of Facile Metal-Carboxylate Displacement and Binding. J. Am. Chem. Soc. 2013, 135, 1853618548 .

(25) Grisorio, R.; Debellis, D.; Suranna, G. P.; Gigli, G.; Giansante, C. The Dynamic Organic/Inorganic Interface of Colloidal PbS Quantum Dots. Angew. Chem., Int. Ed. 2016, 55, 6628-6633.

(26) Nag, A.; Kovalenko, M. V.; Lee, J.; Liu, W.; Spokoyny, B.; Talapin, D. V. Metal-Free Inorganic Ligands for Colloidal Nanocrystals: S2-, HS-, Se2-, HSe-, Te2-, HTe-, TeS32-, OH-, and NH2- as Surface Ligands. J. Am. Chem. Soc. 2011, 133, 10612-10620.

(27) Campos, M. P.; Hendricks, M. P.; Beecher, A. N.; Walravens, W.; Swain, R. A.; Cleveland, G. T.; Hens, Z.; Sfeir, M. Y.; Owen, J. S. A Library of Selenourea Precursors to PbSe Nanocrystals with Size Distributions Near the Homogeneous Limit. J. Am. Chem. Soc. 2017, 139, 2296-2305.

(28) Cass, L. C.; Malicki, M.; Weiss, E. a. The Chemical Environments of Oleate Species within Samples of Oleate-Coated PbS Quantum Dots. Anal. Chem. 2013, 85, 6974-6979.

(29) Mesubi, M. A. An Infrared Study of Zinc, Cadmium, and Lead Salts of Some Fatty Acids. J. Mol. Struct. 1982, 81, 61-71.

(30) Deacon, G. B.; Philiips, R. J. Relationships between the CarbonOxygen Stretching Frequencies of Carboxylato Complexes and the Type of Carboxylate Coordination. Coord. Chem. Rev. 1980, 33, 227250.

(31) Ellis, H. A.; White, N. A. S.; Taylor, R. A.; Maragh, P. T. Infrared, X-Ray and Microscopic Studies on the Room Temperature Structure of Anhydrous Lead (II) N-Alkanoates. J. Mol. Struct. 2005, $738,205-210$.
(32) Moreels, I.; Fritzinger, B.; Martins, J. C.; Hens, Z. Surface Chemistry of Colloidal PbSe Nanocrystals. J. Am. Chem. Soc. 2008, 130, 15081-15086.

(33) Van Aert, S.; Batenburg, K. J.; Rossell, M. D.; Erni, R.; Van Tendeloo, G. Three-Dimensional Atomic Imaging of Crystalline Nanoparticles. Nature 2011, 470 (7334), 374-377.

(34) Scott, M. C.; Chen, C.-C.; Mecklenburg, M.; Zhu, C.; Xu, R.; Ercius, P.; Dahmen, U.; Regan, B. C.; Miao, J. Electron Tomography at 2.4-Ångström Resolution. Nature 2012, 483, 444-447.

(35) Goris, B.; Bals, S.; Van den Broek, W.; Carbó-Argibay, E.; Gómez-Graña, S.; Liz-Marzán, L. M.; Van Tendeloo, G. Atomic-Scale Determination of Surface Facets in Gold Nanorods. Nat. Mater. 2012, 11, 930-935.

(36) Bals, S.; Van Aert, S.; Romero, C. P.; Lauwaet, K.; Van Bael, M. J.; Schoeters, B.; Partoens, B.; Yucelen, E.; Lievens, P.; Van Tendeloo, G. Atomic Scale Dynamics of Ultrasmall Germanium Clusters. Nat. Commun. 2012, 3, 897.

(37) Jones, L.; Macarthur, K. E.; Fauske, V. T.; van Helvoort, A. T. J.; Nellist, P. D. Rapid Estimation of Catalyst Nanoparticle Morphology and Atomic- Coordination by High-Resolution Z-Contrast Electron Microscopy. Nano Lett. 2014, 14, 6336-6341.

(38) Geuchies, J. J.; van Overbeek, C.; Evers, W. H.; Goris, B.; de Backer, A.; Gantapara, A. P.; Rabouw, F. T.; Hilhorst, J.; Peters, J. L.; Konovalov, O.; Petukhov, A. V.; Dijkstra, M.; Siebbeles, L. D. A.; van Aert, S.; Bals, S.; Vanmaekelbergh, D. In Situ Study of the Formation Mechanism of Two-Dimensional Superlattices from PbSe Nanocrystals. Nat. Mater. 2016, 15, 1248-1254.

(39) Green, M. L. H. A New Approach to the Formal Classification of Covalent Compounds of the Elements. J. Organomet. Chem. 1995, 500, 127-148.

(40) Grodzinska, D.; Pietra, F.; van Huis, M. A.; Vanmaekelbergh, D.; de Mello Donega, C. Thermally Induced Atomic Reconstruction of $\mathrm{PbSe} / \mathrm{CdSe}$ Core/shell Quantum Dots into $\mathrm{PbSe} / \mathrm{CdSe} \mathrm{Bi}-\mathrm{Hemi}-$ sphere Hetero-Nanocrystals. J. Mater. Chem. 2011, 21, 11556-11565. 